\title{
Evaluation of right ventricular function and driving pressure with blood gas analysis could better select patients eligible for VV ECMO in severe ARDS
}

\author{
Matthieu Petit ${ }^{1,2^{*}}$, Armand Mekontso-Dessap ${ }^{3,4,5}$, Paul Masi ${ }^{3,4}$, Annick Legras ${ }^{7}$, Philippe Vignon ${ }^{6}$ and \\ Antoine Vieillard-Baron ${ }^{1,2}$
}

Keywords: Acute Respiratory Distress Syndrome, ECMO, Acute cor pulmonale

\section{To the Editor,}

The Acute Respiratory Distress Syndrome (ARDS) is still associated with high mortality [1], despite application of recent guidelines $[2,3]$. The EOLIA study suggested that Extra-Corporeal Membrane Oxygenation (ECMO) could be effective in some of the most severe patients, but failed to demonstrate a $20 \%$ increase in survival [4]. One reason could be that criteria for selecting patients were only based on blood gas analysis. Our hypothesis is that adding other factors could allow a better selection of patients who could benefit from ECMO.

We took advantages to have a large multicentric cohort of patients under protective ventilation for moderate-tosevere ARDS [5] to determine the incidence, characteristics and outcome of patients eligible for ECMO according to EOLIA-based criteria and to identify patients who would benefit the most of the technique. ECMO was only used in these centers as a recue therapy.

Mechanical ventilation was applied in the volumeassist control mode, with a target tidal volume (TV) of 6-8 $\mathrm{mL} / \mathrm{kg}$ (predicted body weight) and a plateau pressure $<30 \mathrm{cmH}_{2} \mathrm{O}$. Respiratory rate could be increased in case of high arterial carbon dioxide partial pressure

${ }^{*}$ Correspondence: matthieu.petit@aphp.fr

${ }^{1}$ Medical Intensive Care Unit, APHP, Ambroise Paré Hospital, Boulogne-Billancourt, France

Full list of author information is available at the end of the article
$\left(\mathrm{PaCO}_{2}\right)$. Prone positioning was left to the discretion of the attending physician, but was typically performed in patients with a $\mathrm{PaO}_{2} / \mathrm{FiO}_{2}<100 \mathrm{mmHg}$ and/or an acute core pulmonale (ACP) [6]. Patients eligible for ECMO according to EOLIA-based criteria were identified as follows: $\mathrm{PaO}_{2} / \mathrm{FiO}_{2}<80 \mathrm{mmHg}$ with optimal PEEP, or a $\mathrm{pH}<7.25$ and $\mathrm{PaCO}_{2}>60 \mathrm{mmHg}$ with a respiratory rate $\geq 35$ cycles $/ \mathrm{min}$, despite the use of prone positioning or nitric oxide inhalation.

Statistical analysis was performed with R.4.0.4. Patients eligible for ECMO were compared to the rest of the cohort. Continuous data, expressed as medians (interquartile ranges), were compared with Mann-Whitney test. Categorical variables, expressed as numbers and percentages, were compared using the chi-square test or Fisher exact test. To evaluate independent factors associated with ICU mortality in this identified subgroup of patients, significant or marginally significant $(p<0.10)$ bivariate risk factors were examined using univariate and multivariable backward stepwise mixed logistic regression stratified on the center. SAPS II was forced in the model.

752 patients were studied. Characteristics and outcome are given in the Table 1. $67(9 \%)$ patients were potentially eligible for ECMO. They had lower $\mathrm{PaO}_{2} /$ $\mathrm{FiO}_{2}(62$ [55-72] versus 114 [90-120] mmHg: $p<0.01)$ and higher incidence of ACP $(42 \%$ versus $20 \%$, $p<0.001)$. Only 8 of them underwent the procedure. original author(s) and the source, provide a link to the Creative Commons licence, and indicate if changes were made. The images or other third party material in this article are included in the article's Creative Commons licence, unless indicated otherwise in a credit line to the material. If material is not included in the article's Creative Commons licence and your intended use is not permitted by statutory regulation or exceeds the permitted use, you will need to obtain permission directly from the copyright holder. To view a copy of this licence, visit http://creativecommons.org/licenses/by/4.0/. The Creative Commons Public Domain Dedication waiver (http://creativeco mmons.org/publicdomain/zero/1.0/) applies to the data made available in this article, unless otherwise stated in a credit line to the data. 
Table 1 Clinical characteristics and outcome of the entire cohort according to EOLIA criteria

\begin{tabular}{|c|c|c|c|}
\hline Characteristics, outcomes and complications & $\begin{array}{l}\text { ARDS patients without EOLIA } \\
\text { criteria } \\
(n=685)\end{array}$ & $\begin{array}{l}\text { ARDS patients with EOLIA } \\
\text { criteria } \\
(n=67)\end{array}$ & $p$-value \\
\hline Age (years) & $59(47-72)$ & $56(47-70)$ & 0.43 \\
\hline Male sex, $n(\%)$ & $465(68)$ & $42(63)$ & 0.47 \\
\hline SAPS $\|$ & $51(38-65)$ & $47(33-64)$ & 0.27 \\
\hline Weight (kg) & $77(65-84)$ & $70(59-86)$ & 0.43 \\
\hline Cause of ARDS, $n(\%)$ & & & 0.20 \\
\hline Pneumonia & $83(12)$ & $8(12)$ & \\
\hline Aspiration & $259(39)$ & $34(51))$ & \\
\hline Non-pulmonary sepsis & $245(37)$ & $17(26)$ & \\
\hline Other causes & $83(12)$ & $7(11)$ & \\
\hline \multicolumn{4}{|l|}{ Respiratory setting at inclusion } \\
\hline Tidal volume (ml/kg) & $6.7(6.0-8.0)$ & $6.02(5.4-6.9)$ & $<0.01$ \\
\hline Respiratory rate (cycle/min) & $22(16-27)$ & $26(22-30)$ & $<0.01$ \\
\hline $\operatorname{PEEP}\left(\mathrm{cmH}_{2} \mathrm{O}\right)$ & $8(5-10)$ & $10(7-12)$ & $<0.01$ \\
\hline Plateau pressure $\left(\mathrm{cmH}_{2} \mathrm{O}\right)$ & $24(21-28)$ & $27(25-29)$ & $<0.01$ \\
\hline Compliance $\left(\mathrm{ml} / \mathrm{cmH}_{2} \mathrm{O}\right)$ & $30.7(24-39.1)$ & $25.9(19.3-33.8)$ & $<0.01$ \\
\hline Driving pressure $\left(\mathrm{cmH}_{2} \mathrm{O}\right)$ & $15(13-19)$ & $17(14-19.8)$ & 0.04 \\
\hline \multicolumn{4}{|l|}{ Arterial blood gases } \\
\hline $\mathrm{PaO}_{2} / \mathrm{FiO}_{2}$ ratio $(\mathrm{mmHg})$ & $114(90-120)$ & $62(55-72)$ & $<0.01$ \\
\hline $\mathrm{PaCO}_{2}(\mathrm{mmHg})$ & $44(38-52)$ & $48(41-60)$ & $<0.01$ \\
\hline Shock, n (\%) & $449(66)$ & $53(79)$ & 0.04 \\
\hline Prone positioning, $n(\%)$ & $163(24)$ & $55(82)$ & $<0.01$ \\
\hline WV ECMO in rescue during ARDS course, $n(\%)$ & $0(0)$ & $8(12)$ & $<0.01$ \\
\hline RRT during ARDS course, $n(\%)$ & $126(30)$ & $15(40)$ & 0.27 \\
\hline \multicolumn{4}{|l|}{ Echocardiographic findings } \\
\hline RVEDA/LVEDA & $0.68(0.57-0.81)$ & $0.83(0.64-1.04)$ & $<0.01$ \\
\hline Systolic pulmonary artery pressure $(\mathrm{mmHg})$ & $35(20-48)$ & $52(35-59)$ & $<0.01$ \\
\hline Severe acute cor pulmonale & $43(6)$ & $11(16)$ & $<0.01$ \\
\hline \multicolumn{4}{|l|}{ Outcome, $n(\%)$} \\
\hline ICU mortality & $243(36)$ & $31(46)$ & 0.10 \\
\hline ICU stay (days) & $16(8-30)$ & $15(6-31)$ & 0.43 \\
\hline
\end{tabular}

Values are expressed as median (interquartile range) or $n$ (\%)

ARDS, Acute Respiratory Distress Syndrome; PEEP, Positive End-Expiratory Pressure; VV ECMO, Veno-Venous Extracorporeal Membrane Oxygenation; RRT, Renal Remplacement Therapy; RVEDA, Right Ventricular End-Diastolic Area; LVDEA, Left Ventricular End-Diastolic Area; ICU, Intensive Care Unit

In-ICU mortality in the whole cohort was $36 \%$. Causes of death in patients eligible for ECMO was multi-organ failure in $21(68 \%)$, neurologic in $4(13 \%)$ and ECMO complication in $3(10 \%)$. Only 3 patients $(10 \%)$ died from hypoxic cardiac arrest.

Characteristics and outcome of patients potentially eligible for ECMO according to ICU mortality are given in the Table 2. In multivariable analysis, severe right ventricular dilatation (right-to-left ventricle enddiastolic area ratio $>1$ ) and driving pressure were the only factors associated with in-ICU mortality (OR [95\% CI]: 5.62 [1.44-27.39], $p=0.02$ and 1.14 [1.01-1.31], $p=0.04$, respectively).
A limitation of our study is that eight patients of the cohort received ECMO as a rescue therapy, which may have influenced our results, especially since the technique is now safer when performed in expert centers. However, six of these eight patients died.

In conclusion, we report a $9 \%$ incidence of patients who reach the EOLIA-based criteria for ECMO in a large non-selected cohort of ARDS patients ventilated with moderate-to-severe ARDS. These patients exhibited higher driving pressure and more frequent right ventricle failure, both being independently associated with ICU mortality. How this subgroup of patients could be considered as the ideal target for ECMO 
Table 2 Clinical characteristics and echocardiographic findings of ARDS patients eligible for ECMO

\begin{tabular}{|c|c|c|c|}
\hline Characteristics, outcomes and complications & $\begin{array}{l}\text { Survivors } \\
(n=36)\end{array}$ & $\begin{array}{l}\text { Non survivors } \\
(n=31)\end{array}$ & $p$-value \\
\hline Age (years) & $56(45-70)$ & $57(48-70)$ & 0.44 \\
\hline Male sex, $n(\%)$ & $23(64)$ & $19(61)$ & 0.47 \\
\hline SAPS $\|$ & $44(32-58)$ & $53(36-75)$ & 0.15 \\
\hline Weight (kg) & $74(63-97)$ & $67(56-80)$ & 0.22 \\
\hline Cause of ARDS, $n(\%)$ & & & 0.63 \\
\hline Pneumonia & $6(17)$ & $2(7)$ & \\
\hline Aspiration & $18(50))$ & $16(53)$ & \\
\hline Non-pulmonary sepsis & $8(22)$ & $9(30)$ & \\
\hline Other causes & $4(11)$ & $3(10)$ & \\
\hline \multicolumn{4}{|l|}{ Respiratory setting at inclusion } \\
\hline Tidal volume (ml/kg) & $6.3(5.5-7.6)$ & $5.9(5.3-6.6)$ & 0.13 \\
\hline Respiratory rate (cycle/min) & $25(20-27)$ & $30(25-30)$ & $<0.01$ \\
\hline $\operatorname{PEEP}\left(\mathrm{cmH}_{2} \mathrm{O}\right)$ & $10(8-12)$ & $10(7-12)$ & 0.72 \\
\hline Plateau pressure $\left(\mathrm{cmH}_{2} \mathrm{O}\right)$ & $26(24-29)$ & $28(25-38)$ & 0.07 \\
\hline Compliance $\left(\mathrm{ml} / \mathrm{cmH}_{2} \mathrm{O}\right)$ & $30(2338)$ & $23(16-28)$ & $<0.01$ \\
\hline Driving pressure $\left(\mathrm{cmH}_{2} \mathrm{O}\right)$ & $16(14-19)$ & $19(16-22)$ & 0.05 \\
\hline \multicolumn{4}{|l|}{ Arterial blood gases } \\
\hline $\mathrm{PaO}_{2} / \mathrm{FiO}_{2}$ ratio $(\mathrm{mmHg})$ & $69(58-74)$ & $60(55-67)$ & 0.08 \\
\hline $\mathrm{PaCO}_{2}(\mathrm{mmHg})$ & $48(40-52)$ & $51(42-70)$ & 0.17 \\
\hline Shock, n (\%) & $25(70)$ & $28(90)$ & 0.07 \\
\hline Prone positioning, $n(\%)$ & $31(86)$ & $24(77)$ & 0.52 \\
\hline WV ECMO in rescue during ARDS course, $n(\%)$ & $2(1)$ & $6(19)$ & 0.13 \\
\hline RRT during ARDS course, $n(\%)$ & $9(33)$ & $6(55)$ & 0.28 \\
\hline \multicolumn{4}{|l|}{ Echocardiographic findings } \\
\hline RVEDA/LVEDA & $0.71(0.57-0.93)$ & $0.98(0.71-1.10)$ & 0.10 \\
\hline Pulmonary hypertension (mmHg) & $51(44-55)$ & $52(34-63)$ & 0.85 \\
\hline Severe acute cor pulmonale & $3(8)$ & $8(26)$ & 0.09 \\
\hline ICU stay (days) & $19(14-34)$ & $12(3-20)$ & 0.16 \\
\hline
\end{tabular}

Values are expressed as median (interquartile range) or $n$ (\%)

ARDS, Acute Respiratory Distress Syndrome; PEEP, Positive End-Expiratory Pressure; VV ECMO, Veno-Venous Extracorporeal Membrane Oxygenation; RRT, Renal Remplacement Therapy; RVEDA, Right Ventricular End-Diastolic Area; LVDEA, Left Ventricular End-Diastolic Area; ICU, Intensive Care Unit

selection strategy should better be evaluated in the future.

\section{Abbreviations}

ARDS: Acute Respiratory Distress Syndrome; ECMO: Extra-Corporeal Membrane Oxygenation; TV: Tidal Volume; ACP: Acute Core Pulmonale; PEEP: Positive End Expiratory Pressure; SAPS II: Simplified Acute Physiology Score II; ICU: Intensive Care Unit; RRT: Renal Replacement Therapy; RVEDA: Right Ventricular End-Diastolic Area; LVDEA: Left Ventricular End-Diastolic Area.

\section{Acknowledgements}

Not applicable.

\section{Authors' contributions}

MP and AVB designed the study; AMD, PV, PM, AL collected the data, MP did the statistical analysis, MP and AVB wrote the manuscript; all authors reviewed the manuscript. All authors read and approved the final manuscript.

\section{Funding \\ None.}

\section{Availability of data and materials}

The datasets used and/or analysed during the current study are available from the corresponding author on reasonable request.

\section{Declaration}

Ethics approval and consent to participate

The study was approved by the Institutional Review Boards of participating centers as a component of standard of care and requirement for patient's consent was waived.

\section{Consent for publication}

Not applicable.

\section{Competing interests}

MP, PM, AL, PV declare no competing interest. AMD reports grants from Fischer Paykel, Baxter, and Ferring, and personal fees from Air Liquide, Amomed, 
and Addmedica, all outside the submitted work. AVB reports research Grant from GSK, outside the submitted work.

\section{Author details}

${ }^{1}$ Medical Intensive Care Unit, APHP, Ambroise Paré Hospital, Boulogne-Billancourt, France. ${ }^{2}$ INSERM UMR 1018, Clinical Epidemiology Team, CESP, Université de Paris Saclay, Villejuif, France. ${ }^{3}$ Medical Intensive Care Unit, APHP, HenriMondor Hospital, 94010 Créteil, France. ${ }^{4}$ CARMAS, Paris Est Créteil University, 94010 Créteil, France. ${ }^{5}$ INSERM, IMRB, Paris Est Créteil University, 94010 Créteil, France. ${ }^{6}$ Medical-Surgical Intensive Care Unit and CIC 1435, Limoges University Hospital, 87000 Limoges, France. ${ }^{7}$ Medical Intensive Care Unit, Tours University Hospital, Tours, France.

Received: 17 June 2021 Accepted: 18 June 2021

Published online: 27 June 2021

\section{References}

1. Bellani G, Laffey JG, Pham T, Fan E, Brochard L, Esteban A, et al. Epidemiology, patterns of care, and mortality for patients with acute respiratory distress syndrome in intensive care units in 50 countries. JAMA. 2016;315(8):788-800.
2. Fan E, Del Sorbo L, Goligher EC, Hodgson CL, Munshi L, Walkey AJ, et al. An official American Thoracic Society/European Society of Intensive Care Medicine/Society of Critical Care Medicine clinical practice guideline: mechanical ventilation in adult patients with acute respiratory distress syndrome. Am J Respir Crit Care Med. 2017;195(9):1253-63.

3. Papazian L, Aubron C, Brochard L, Chiche J-D, Combes A, Dreyfuss D, et al. Formal guidelines: management of acute respiratory distress syndrome. Ann Intensive Care. 2019;9(1):69.

4. Combes A, Hajage D, Capellier G, Demoule A, Lavoué S, Guervilly C, et al. Extracorporeal membrane oxygenation for severe acute respiratory distress syndrome. N Engl J Med. 2018;378(21):1965-75.

5. Mekontso Dessap A, Boissier F, Charron C, Bégot E, Repessé X, Legras $A$, et al. Acute cor pulmonale during protective ventilation for acute respiratory distress syndrome: prevalence, predictors, and clinical impact. Intensive Care Med. 2016;42(5):862-70.

6. Vieillard-Baron A, Prin S, Chergui K, Dubourg O, Jardin F. Echo-Doppler demonstration of acute cor pulmonale at the bedside in the medical intensive care unit. Am J Respir Crit Care Med. 2002;166(10):1310-9.

\section{Publisher's Note}

Springer Nature remains neutral with regard to jurisdictional claims in published maps and institutional affiliations.
Ready to submit your research? Choose BMC and benefit from:

- fast, convenient online submission

- thorough peer review by experienced researchers in your field

- rapid publication on acceptance

- support for research data, including large and complex data types

- gold Open Access which fosters wider collaboration and increased citations

- maximum visibility for your research: over $100 \mathrm{M}$ website views per year

At BMC, research is always in progress.

Learn more biomedcentral.com/submissions 